\title{
Development of explicit criteria for cholecystectomy
}

\author{
J M Quintana, J Cabriada, I López de Tejada, M Varona, V Oribe, B Barrios, \\ I Aróstegui, A Bilbao
}

See end of article for authors' affiliations

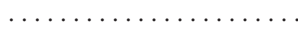

Correspondence to: Dr J M Quintana, Unidad de Investigación, Hospital de Galdakao, Barrio Labeaga s/n, 48960 Galdakao, Vizcaya, Spain; jmquinta@

hgda.osakidetza.net

Accepted for publication 9 May 2002
Objective: Consensus development techniques were used in the late 1980s to create explicit criteria for the appropriateness of cholecystectomy. New diagnostic and treatment techniques have been developed in the last decade, so an updated appropriateness of indications tool was developed for cholecystectomy in patients with non-malignant diseases. The validity and reliability of panel results using this tool were tested.

Methods: Criteria were developed using a modified Delphi panel judgement process. The level of agreement between the panellists (six gastroenterologists and six surgeons) was analysed and the ratings were compared with those of a second different panel using weighted kappa statistics.

Results: The results of the main panel were presented as a decision tree. Of the 210 scenarios evaluated by the main panel in the second round, $51 \%$ were found appropriate, $26 \%$ uncertain, and $23 \%$ inappropriate. Agreement was achieved in $54 \%$ of the scenarios and disagreement in $3 \%$. Although the gastroenterologists tended to score fewer scenarios as appropriate, as a group they did not differ from the surgeons. Comparison of the ratings of the main panel with those of a second panel resulted in a weighted kappa statistic of 0.75 .

Conclusions: The parameters tested showed acceptable validity and reliability results for an evaluation tool. These results support the use of this algorithm as a screening tool for assessing the appropriateness of cholecystectomy.
G allstones are a common problem in developed countries. ${ }^{1}$ Cholecystectomy is the procedure used most frequently to treat gallstones, ${ }^{23}$ although there are variations in its use in different geographical areas. ${ }^{45}$

Inexplicable variations in surgical rates, identification of inappropriate care, and escalating healthcare costs raise questions about potential underuse or overuse of many medical and surgical procedures, ${ }^{6}$ including cholecystectomy. Central to this question is the determination of what constitutes appropriate indications for a given procedure. Unfortunately, there are no rigorous scientific data on efficacy and effectiveness to justify medical practice, so other factors must be used to determine criteria for appropriateness. ${ }^{78}$ Uncertainty as to the indications for cholecystectomy has been reported by a number of researchers-for example, patients with an asymptomatic calculus, ${ }^{9}$ those with symptoms suggestive of cholelithiasis but no diagnostic evidence, ${ }^{10}$ and those with polyps or cholesterolosis. ${ }^{1}$

A method that combines expert opinion with available scientific evidence developed by investigators at the RAND Corporation and the University of California at Los Angeles ${ }^{12}$ has been used to evaluate the appropriateness of a number of medical and surgical interventions. Previous studies of cholecystectomy using the RAND methodology were performed in the early 1980s in the US ${ }^{12}$ and in the late 1980s in Israel ${ }^{13}$ and the $\mathrm{UK}^{14}$ to provide managers and clinicians with information that would help them decide whether or not cholecystectomy was an appropriate treatment for a specific patient. Important changes have since occurred in this field-for example, the introduction of echography and other imaging tests has improved the diagnosis of patients with gallbladder disease or digestive symptoms and new pharmacological and invasive modalities such as endoscopic retrograde cholangiopancreatography (ERCP) and surgical procedures such as laparoscopy have changed the indications for cholecystectomy. The criteria developed in the 1980s are therefore no longer useful.

The validity of any tool is important. Studies to date have investigated the relation between the literature and the ratings ${ }^{15}$ the reliability of the ratings, ${ }^{16}$ and the face, content, and construct validity. ${ }^{17}{ }^{18}$ Despite this, criticisms of the appropriateness method include the low sensitivity of the results to changes over time, to the selection of experts, ${ }_{19}^{19}$ to the composition of the panel, ${ }^{20}{ }^{21}$ and to the validity and reliability of the results. $^{22}$

This study was undertaken to provide an updated review of the indications for cholecystectomy in patients with nonmalignant disease using the RAND methodology and to study its validity and reliability.

\section{METHODS}

\section{Explicit criteria development}

The criteria for measuring the appropriateness of the use of cholecystectomy were developed using a previously described explicit method-the RAND appropriateness method ${ }^{23}$-as follows. An extensive literature review was performed to summarize existing knowledge concerning the efficacy, effectiveness, risks, costs, and opinions about the use of cholecystectomy to treat non-malignant diseases. From this review a comprehensive and detailed list of mutually exclusive and clinically specific scenarios (indications) was developed in which cholecystectomy might be performed. This list contained 210 indications for cholecystectomy in patients with no special circumstances such as pregnancy or comorbidities such as diabetes or immunosuppression, which were considered separately. Each indication was specified in sufficient detail that patients within a given indication were reasonably homogeneous.

The indications for cholecystectomy included the following variables:

- Patients with symptomatic cholelithiasis without complications: age $\left(<76\right.$ years and $>75$ years ${ }^{24} 25$ ), gallbladder and common bile duct imaging studies (macrolithiasis or microlithiasis, thickened gallbladder, dilated bile duct), surgical risk (low or high, based on the American Society of Anesthesiologists [ASA] criteria $^{26}$ ). 


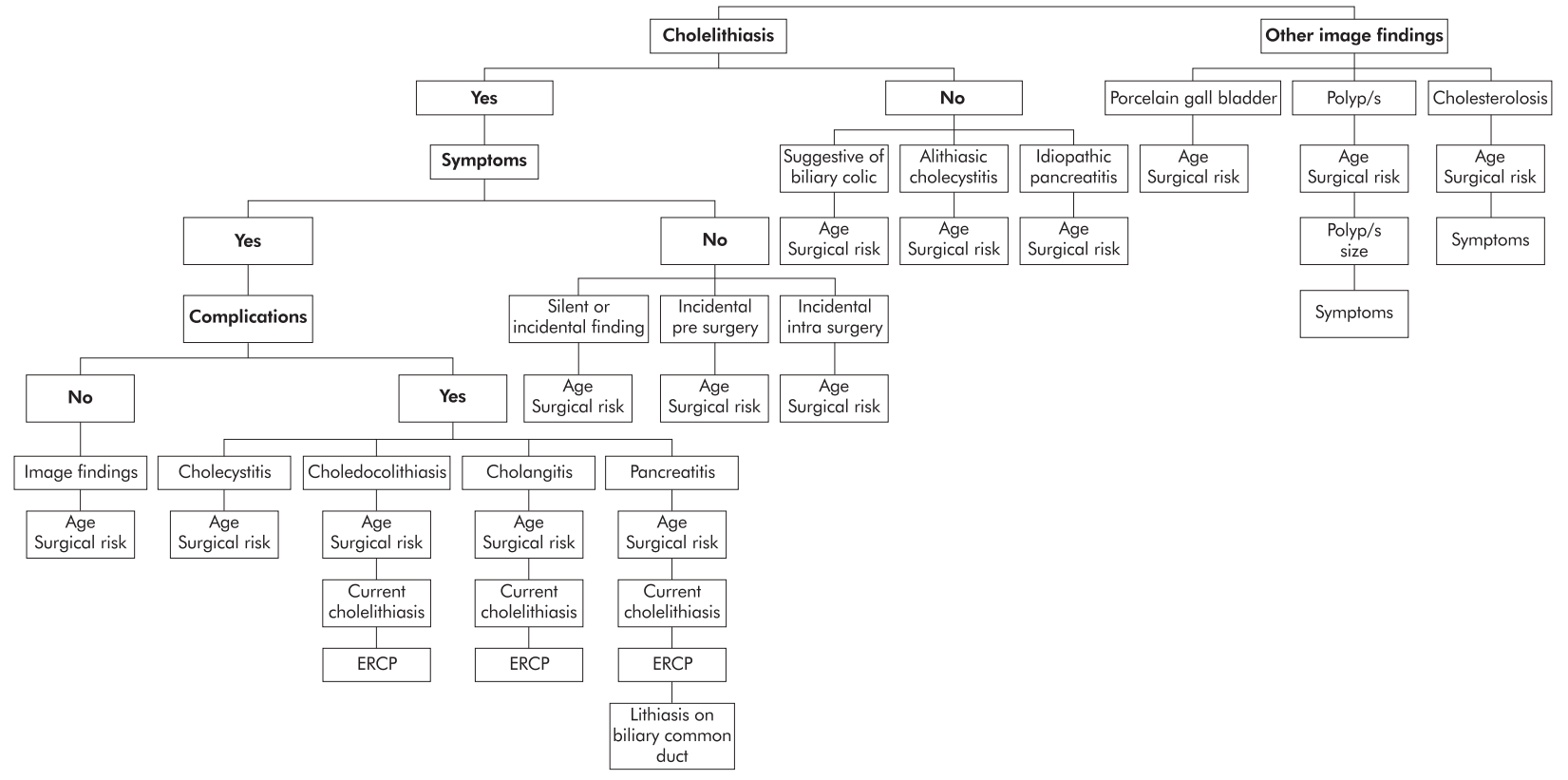

Figure 1 Variables included in the algorithm and their main categories.

- Patients with complications: if cholecystitis, age and surgical risk; if choledocholithiasis, age, surgical risk, presence of current cholelithiasis and previous non-surgical procedures performed as ERCP (successful or not); if cholangitis, same as above; if pancreatitis, same as above plus presence of current lithiasis on common bile duct (yes or no).

- Asymptomatic patients with cholelithiasis: four clinical situations-silent calculus, incidental finding on an imaging test, incidental findings before a surgical intervention in the area, or incidental findings within a surgical intervention in the area. The variables included were age, surgical risk, and previous surgical procedures performed in the area (yes or no).

- Patients with symptoms suggestive of cholelithiasis without cholelithiasis: symptoms suggestive of biliary colic, alithiasic cholecystitis, and idiopathic pancreatitis. Age and surgical risk were included in all.

- Miscellaneous category that included imaging findings: porcelain gallbladder, polyps $(\geqslant 1 \mathrm{~cm}$ or $<1 \mathrm{~cm}$ ), and cholesterolosis, the latter two groups with or without the presence of symptoms.

The 210 indications resulted from all possible combinations of the variables described previously and their respective categories. The algorithm is shown in fig 1 .

A national panel of six experienced surgeons and six gastroenterologists was formed (panel 1). Panellists were nationally recognized specialists in the field whose names were provided by their respective medical societies and members of our research team. They were provided with the literature review and the list of indications and were asked to rate each indication for the appropriateness of performing cholecystectomy based on the average patient and average physician in the year 2000. Appropriateness was defined as meaning that the expected health benefit exceeds the expected negative consequences by a sufficiently wide margin to make cholecystectomy worth performing, choosing the best surgical alternative available for the patient (open cholecystectomy, minicholecystectomy, or laparoscopy).

Ratings were scored on a 9 point scale. The use of cholecystectomy for a specific indication was considered appropriate if the panel's median rating was 7-9 without disagreement, inappropriate if the value was $1-3$ without disagreement, or uncertain if the median rating was 4-6 or if the members of the panel disagreed. Disagreement was defined as at least one third of the panellists rating an indication as $1-3$ and at least another third rating it as 7-9. This method did not attempt to force panellists to reach agreement on appropriateness. It was beyond the scope of this study to compare the use of open cholecystectomy with laparoscopic cholecystectomy. The panellists were instructed to evaluate the appropriateness of performing any cholecystectomy technique against other nonsurgical treatments, taking a "watch and wait" strategy, or doing nothing. They were instructed not to evaluate the appropriate timing of the intervention (urgent or as an interval operation) but only to consider whether the intervention itself was appropriate.

The ratings were confidential and took place in two rounds using a modified Delphi process. In the first round the results

Table 1 Agreement and appropriateness judgement of the panel of experts for cholecystectomy

\begin{tabular}{lcccc}
\hline & \multicolumn{2}{l}{ Appropriateness judgement } & \\
\cline { 2 - 4 } Panel agreement & Appropriate & Uncertain & Inappropriate & Total \\
\hline Agreement & $86(80.4 \%)$ & $0(0 \%)$ & $27(56.3 \%)$ & $113(53.8 \%)$ \\
Indeterminate & $21(19.6 \%)$ & $49(89.1 \%)$ & $21(43.7 \%)$ & $91(43.3 \%)$ \\
Disagreement & $0(0 \%)$ & $6(10.9 \%)$ & $0(0 \%)$ & $6(2.9 \%)$ \\
Total & $107(50.9 \%)$ & $55(26.2 \%)$ & $48(22.9 \%)$ & 210 \\
\hline
\end{tabular}

The percentages in parentheses are for indications rated in each category from the total number of indications rated by the main panel in each appropriateness category, with the exception of the total row. 
Table 2 Mean scores, mean deviations, and mean change in the evaluations of the expert panelists in rounds 1 and 2 for cholecystectomy

\begin{tabular}{|c|c|c|c|c|c|c|}
\hline \multirow[b]{2}{*}{ Panelistł } & \multirow[b]{2}{*}{ Specialty $\ddagger$} & \multicolumn{2}{|c|}{ Mean score* } & \multirow{2}{*}{$\begin{array}{l}\text { Mean } \\
\text { change }\end{array}$} & \multicolumn{2}{|c|}{ Mean deviation† } \\
\hline & & Round 1 & Round 2 & & Round 1 & Round 2 \\
\hline$A$ & $\mathrm{~S}$ & 7.1 & 6 & -1.1 & 1.2 & 0.8 \\
\hline B & $S$ & 5.9 & 5.7 & -0.2 & 0.7 & 0.8 \\
\hline C & $\mathrm{S}$ & 6 & 6 & 0 & 1.2 & 1.2 \\
\hline D & $S$ & 5.7 & 5.3 & -0.4 & 1 & 1.6 \\
\hline$E$ & $S$ & 7.1 & 6.5 & -0.6 & 1.6 & 1.1 \\
\hline $\mathrm{F}$ & S & 7.2 & 6.4 & -0.8 & 1.4 & 1.2 \\
\hline G & GE & 3.6 & 5.6 & 2 & 2.5 & 1.8 \\
\hline $\mathrm{H}$ & GE & 6.1 & 6.3 & 0.2 & 1.1 & 0.9 \\
\hline I & GE & 6.3 & 6.6 & 0.3 & 1.2 & 0.9 \\
\hline J & GE & 5.4 & 4.9 & -0.5 & 1.4 & 1.8 \\
\hline K & GE & 4.4 & 5 & 0.6 & 1.7 & 1.2 \\
\hline L & GE & 3.4 & 4.9 & 1.5 & 2.7 & 1.3 \\
\hline
\end{tabular}

* Mean scores are the means of the ratings given by the panelist for all the indications.

tMean deviations are the mean absolute deviation of each panelist from the round 1 median for all indications.

$\ddagger$ The multidisciplinary national panel included six surgeons $(S)$ and six gastroenterologists (GE).

Letters were assigned to each panelist to preserve anonymity.

Table 3 Comparison of the ratings of the two panels

\begin{tabular}{lcccr}
\hline & \multicolumn{2}{l}{ Panel $1^{*}$} & & \\
\cline { 2 - 4 } Panel $2 \dagger$ & Appropriate & Uncertain & Inappropriate & Total \\
\hline Appropriate & $83(77.6 \%)$ & $5(9.1 \%)$ & $0(0 \%)$ & $88(41.9 \%)$ \\
Uncertain & $24(22.4 \%)$ & $42(76.4 \%)$ & $8(16.7 \%)$ & $74(35.2 \%)$ \\
Inappropriate & $0(0 \%)$ & $8(14.5 \%)$ & $40(83.3 \%)$ & $48(22.9 \%)$ \\
Total & $107(50.9 \%)$ & $55(26.2 \%)$ & $48(22.9 \%)$ & 210 \\
\hline
\end{tabular}

The percentages in parentheses are for indications rated in each category from the total of indications rated by panel 1 in each appropriateness category.

*Panel 1 is a multidisciplinary national panel made up of six surgeons and six gastroenterologists.

†Panel 2 is a new panel composed of six different surgeons and six gastroenterologists.

were collated and presented to the panellists at a 1 day meeting. Each panellist received the anonymous ratings of the other panellists as well as a reminder of his own ratings. After extensive discussion, panellists revised the indications according to the definitions. During the first round each panellist rated 192 separate indications and during the second round 210 indications because a new age category was added in some cases.

To study the reliability of panel 1 , a second panel (panel 2) of six surgeons and six gastroenterologists was created by contacting other reputable specialists from different areas throughout the Basque Country. The same documents that were supplied to panel 1 and the 210 original indications were sent to them to be rated in just one round. The final scores were then compared with those of panel 1.

Data relating to some of the algorithm variables were collected for 780 patients from their medical records and the number of theoretical scenarios used was calculated for each of the main diagnostic groups.

\section{Statistical analysis}

For each panellist the mean appropriateness rating of all indications, the percentage of all indications rated for appropriateness as $1-3,4-6$, and 7-9 in all rounds, and the mean change between the two rounds were estimated. Decision algorithms which should permit rapid estimation of appropriateness in practice were compiled from the final results. Weighted kappa statistics ${ }^{27}$ and $95 \%$ confidence intervals (CI) were calculated to test agreement between different panels. All statistical analyses were performed using SAS for Windows version $8 .^{28}$

\section{RESULTS}

\section{Panel results}

After the second round $50.9 \%$ of the 210 scenarios were considered appropriate, $26.2 \%$ uncertain, and $22.9 \%$ inappropriate. Agreement was reached on $53.8 \%$, mainly in the appropriate category ( $80.4 \%$ agreement of all appropriate scenarios, table 1).

Changes in the panellists' scoring from round 1 to 2 were minor, except for those who had the lowest and highest scores during the first round who moved their scores to the group mean in the second round (table 2). No differences were found in the scoring between surgeons and gastroenterologists with mean (SD) scores at the second round of $5.98(0.44)$ and 5.55 (0.75), respectively.

A second panel of six surgeons and six gastroenterologists (panel 2) scored all the indications; the reliability of this panel compared with panel 1 gave a kappa statistic of 0.75 (95\% CI 0.68 to 0.82 ). No disagreement was found between the two panels for appropriate/inappropriate categories, but panel 2 tended to rate fewer indications as appropriate than panel 1 (table 3). The percentage of uncertain indications was higher in panel 2 than in panel 1 , while the percentage of indications considered inappropriate was similar.

\section{Synthesis of panel results}

Five groups of scenarios were analysed (fig 1):

(1) patients with symptomatic cholelithiasis without complications: the panel considered a cholecystectomy to be appropriate in patients with ASA grade I-III; a thickened gallbladder and a dilated common bile duct and age $<76$ years; or an ASA grade IV and age $<76$ years with no previous surgical interventions in the supramesocolic area (fig 2); 


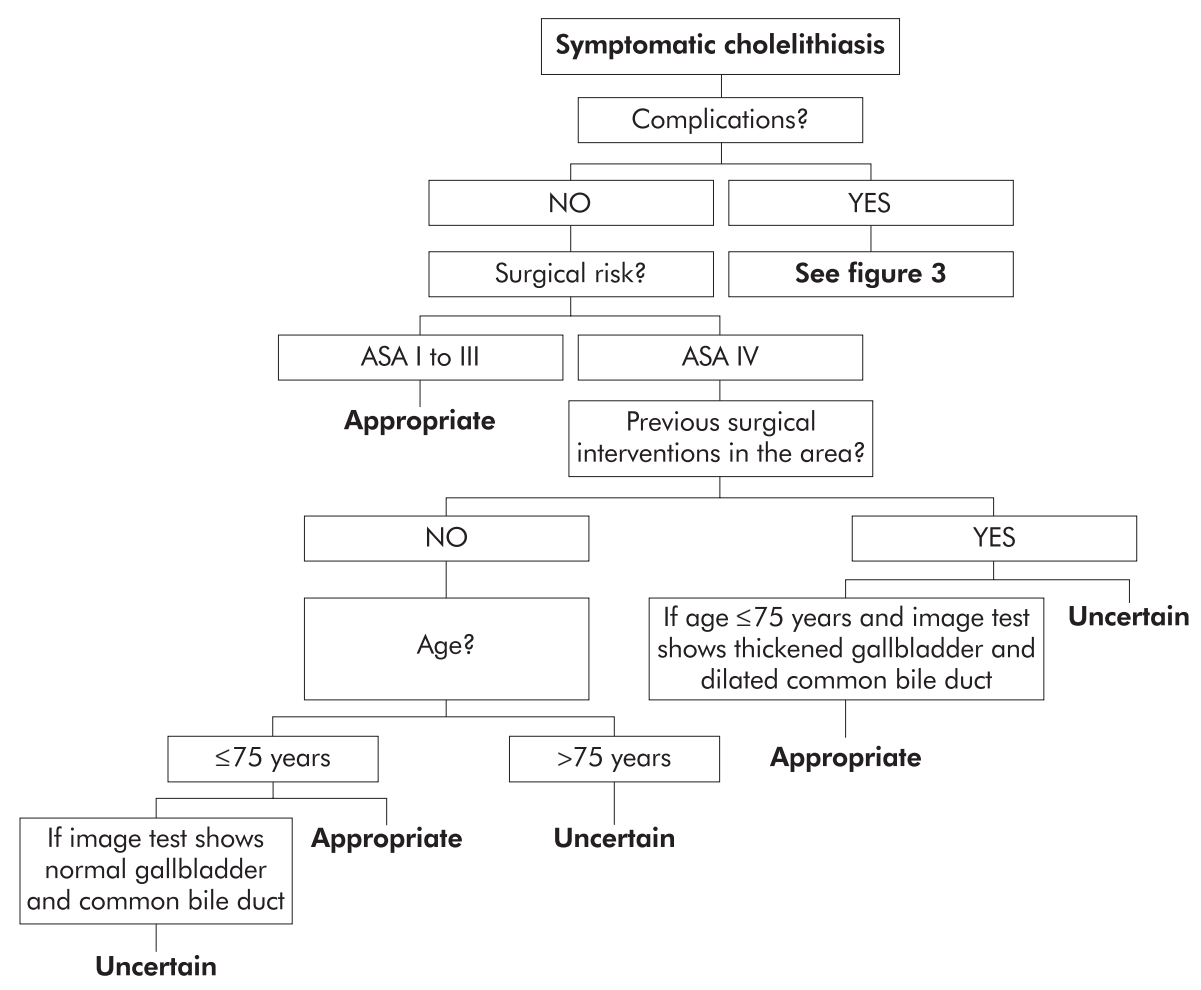

Figure 2 Appropriateness of cholecystectomy in patients with symptomatic cholelithiasis without complications.

(2) patients with symptomatic cholelithiasis with complications which included cholecystitis, pancreatitis, choledocholithiasis, and cholangitis (fig 3):

(3) asymptomatic cholelithiasis (fig 4);

(4) symptoms suggestive of biliary disease without cholelithiasis on imaging: in these patients and in those with a diagnosis of idiopathic pancreatitis cholecystectomy was never an option. In those with a diagnosis of alithiasic cholecystitis aged $<76$ years the intervention was judged to be appropriate (fig 5);

(5) other imaging findings (those related to porcelain gallbladder, polyps, and cholesterolosis) were analysed as separate scenarios (fig 6).

\section{Field study}

Of the 210 theoretical scenarios scored by the panel of experts, only $44(21 \%)$ were used in the 780 medical records reviewed in the field study. Of those with cholelithiasis without complications, nine of 30 scenarios $(30 \%)$ were used for 455 patients $(58.3 \%)$; in those with a complication $(25.8 \%$ of the sample) 22 of 126 theoretical scenarios (17.5\%) were used; seven of 30 scenarios (23.3\%) were asymptomatic ( $12.3 \%$ of patients); $0.5 \%$ of patients had symptoms but no cholelithiasis (2/18 scenarios, $1.1 \%)$; and in the miscellaneous group (cholesterolosis, polyp, or porcelain gallbladder) eight of 42 scenarios (19\%) were used for $3.1 \%$ of patients.

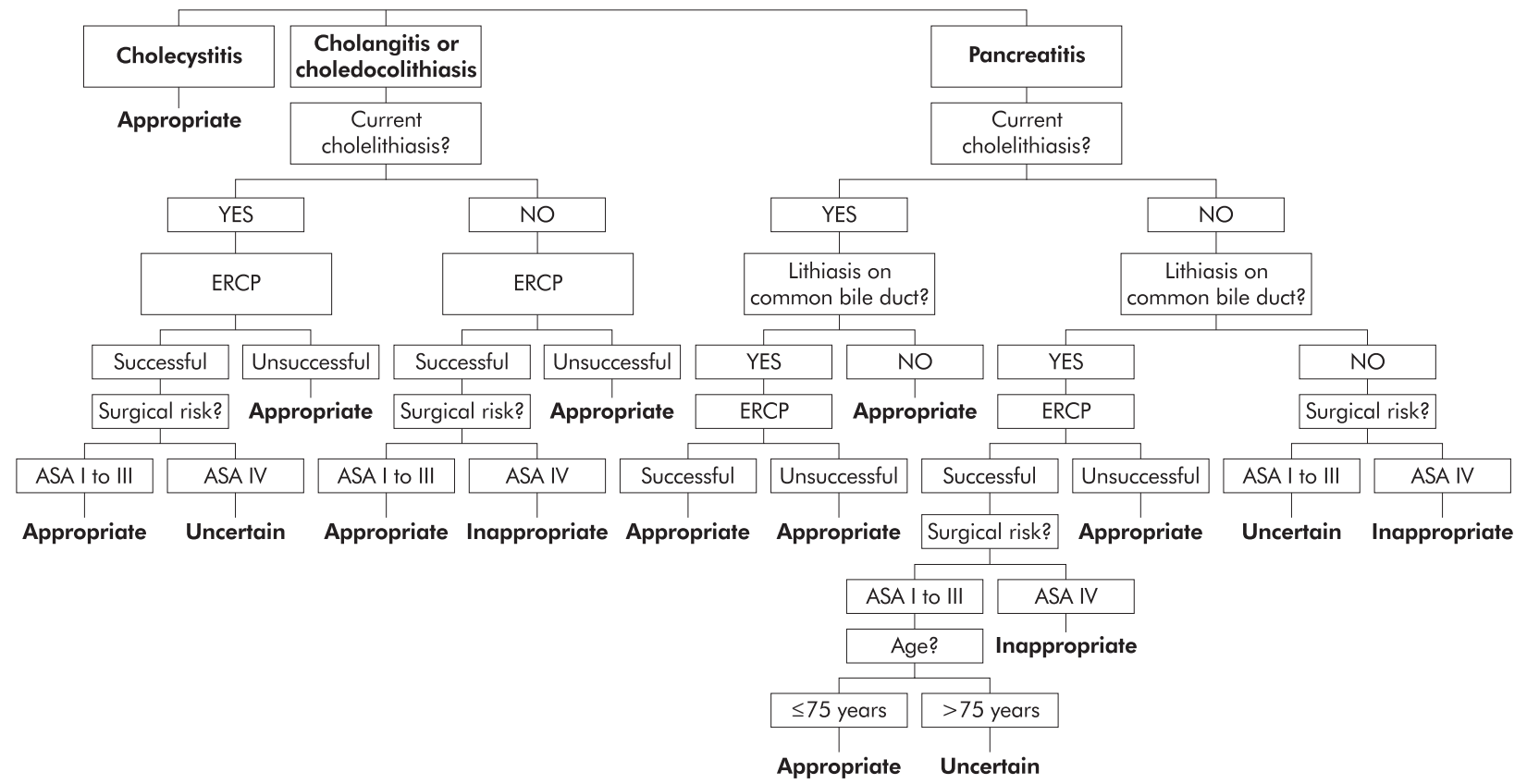

Figure 3 Appropriateness of cholecystectomy in patients with symptomatic cholelithiasis with complications. 


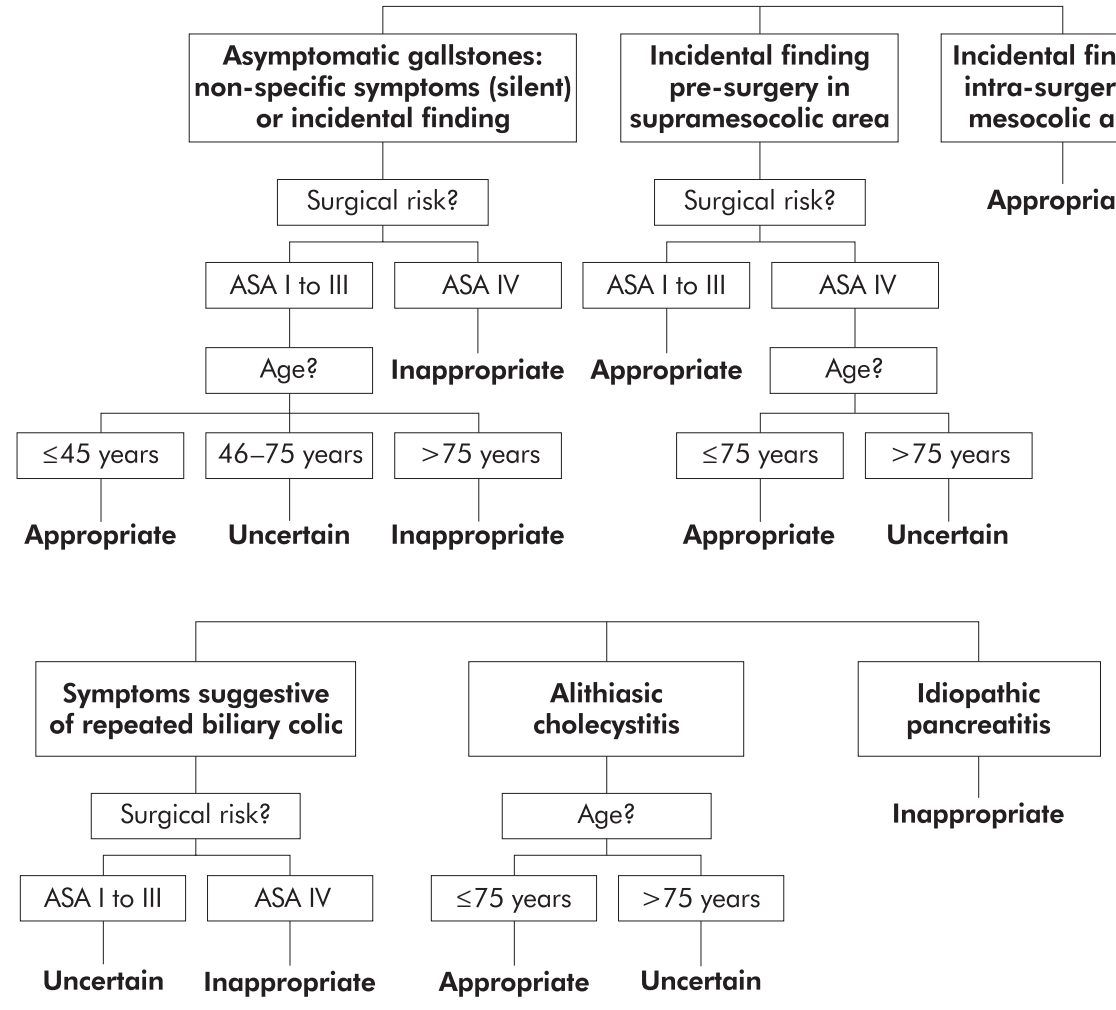

Figure 4 Appropriateness of cholecystectomy in patients with asymptomatic cholelithiasis.

Asymptomatic gallstones: or incidental finding

Age?
Incidental finding

Surgical risk

priate

Figure 5 Appropriateness of cholecystectomy in patients with suggestive symptoms but without cholelithiasis.

Exceptions: (1) If ASA I to III, then Appropriate; (2) If age $\leq 75$ years and ASA I to III, then Uncertain.

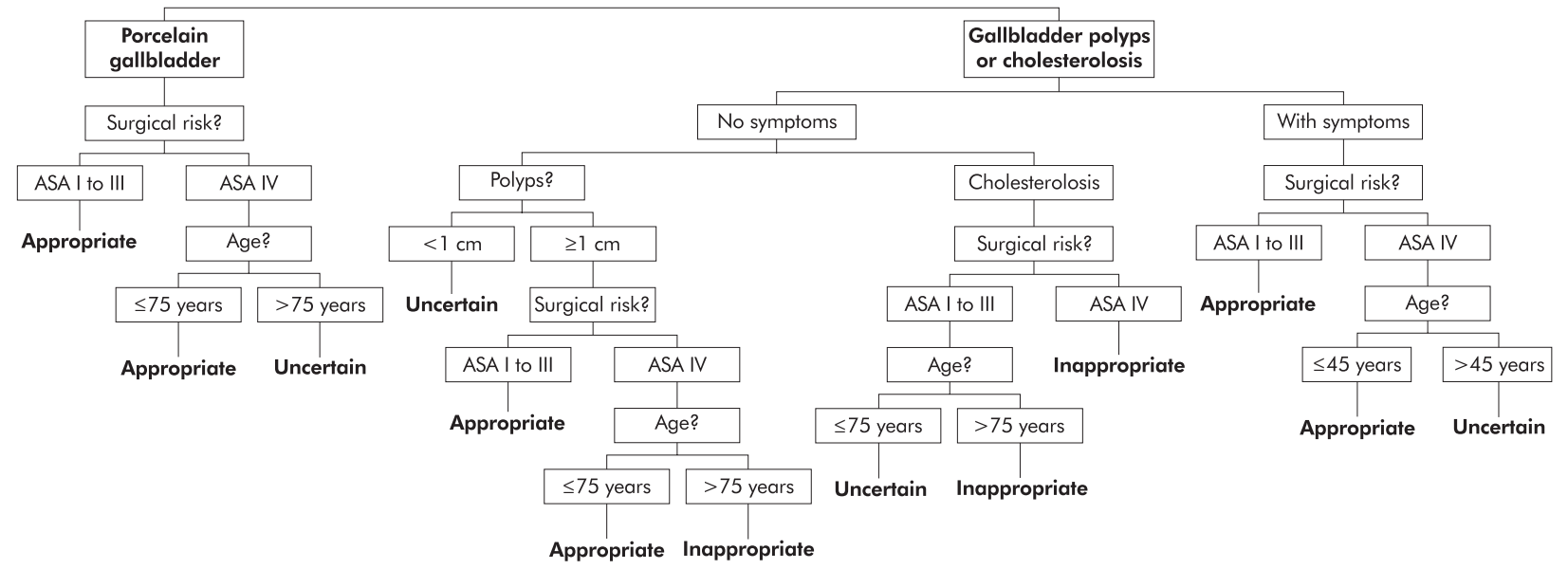

Exceptions: (1) If age $\leq 45$ years and ASA I to III, then Appropriate. If age $>75$ years and ASA IV, then Inappropriate; (2) If previous surgery in the area, then Uncertain.

Figure 6 Appropriateness of cholecystectomy in other specific situations (findings on image tests).

\section{DISCUSSION}

Cholecystectomy has been used by a number of authors to study the variability or the appropriateness of its use. ${ }^{92}$ In this study we have included updated and clear algorithms to provide clinicians with a rapid decision making process for specific patients. The main complaints which can result in cholecystectomy are included in the explicit criteria which were developed using the RAND appropriateness method. Our findings support, in part, the validity and reliability of the tool. The aim is to reduce inappropriate interventions, to increase appropriate procedures, and thus to improve the quality of care provided to patients with gallbladder disease.

The previous RAND cholecystectomy panels used in thel980s in the US ${ }^{12}$ and Israel $^{13}$ included nine expert members (three gastroenterologists, two surgeons, two internists, one family physician, and one radiologist). The UK group $^{14}$ had two panels-one mixed panel with six specialists (two gastroenterologists, one surgeon, one internist, one family physician, and one radiologist) and one single specialist panel with eight surgeons. The US group developed 192 scenarios, the Israeli panel included 11 chapters and 266 scenarios, while the UK had 272 scenarios but shared similar variables. Although the three groups used a similar methodology, they differed slightly in the variables and categories included. The US panel disagreed on $20.4 \%$ of the scenarios and agreed on $53.1 \%$, the Israeli panel disagreed on $35.3 \%$, while the UK mixed panel disagreed on $15 \%$ and the single specialist panel on $8 \%$. The experts on the Israeli panel agreed 
on $46.7 \%$ and those on the two British panels agreed on $67 \%$ and $61 \%$, respectively. Finally, the Israeli experts determined that $47.6 \%$ of the scenarios were appropriate and $46.8 \%$ were inappropriate and in the UK study $13-19 \%$ considered the scenarios to be appropriate and $27-50 \%$ to be inappropriate, depending on which panel criteria were used.

The variables included in the algorithms used in these three studies and ours are not comparable. As the result of technological changes in the last 10-20 years, we introduced variables such as the use of ERCP or new imaging tests such as echography which were not included in the earlier algorithms. We attempted to incorporate new evidence and new scenarios into our algorithm to present updated explicit criteria for the indications for cholecystectomy. An equal number of gastroenterologists and surgeons directly involved in the treatment decision making in Spain were included on the panel.

Our results showed that our main panel reached an acceptable level of agreement after the second round; they were uncertain in $26 \%$ of the theoretical scenarios, which is similar to or lower than in many RAND studies. The reliability of the two panels was good (weighted kappa 0.75), taking into account that panel 2 did the scoring in just one round which would have limited the agreement and created more uncertain scenarios. ${ }^{29}$ As noted elsewhere, the use of a panel of experts has some limitations, including the composition of the panel. In most studies in which mixed panels have been used, clinicians who do not perform a procedure tend to score lower than those who do perform it. ${ }^{2021} 2930$ In our case, although some gastroenterologists scored lower than surgeons, as a group there were no differences between them.

We compared the results of our panel of experts with the judgement of a group of specialists and the evidence in the literature over the last 10 years since the latest RAND panels. Our panel tended not to recommend cholecystectomy mainly for patients with high surgical risk (ASA IV) ${ }^{31}$ and/or older patients and, in cases where applicable, if ERCP was successful. However, there is a lack of evidence as to the appropriate treatment for some common clinical situations such as asymptomatic cholelithiasis and polyps. Even so, our panel criteria matched those reflected in some reports on asymptomatic cholelithiasis, ${ }^{10}$ polyps, ${ }^{11}$ and special clinical situations such as alithiasic cholecystitis. ${ }^{32}$ Most authors tend to recommend no intervention in those with asymptomatic cholelithiasis, although our panel did recommend cholcystectomy for those aged $<45$ years or when the condition was found incidentally. This contradiction probably reflects current clinical practice without any evidence to support the recommendation. The case of those patients with cholelithiasis-like symptoms without lithiasis is controversial except for those with a diagnosis of alithiasic cholecystitis in whom the intervention is recommended. Except for these clinical situations, the criteria of our panel were comparable to those of the current evidence.

Different studies have pointed out the limitations of the RAND method which include lack of evidence in the field, quality of the literature review given to the panel, scope of the definition of appropriateness, panel composition, quality and management of the panel discussion, validity (sensitivity and specificity, predictive validity) and reliability of their conclusions, sensitivity to changes over time, unsolved or uncertain scenarios, and usefulness of their results. ${ }^{15-22}$ We tried to avoid some of these known limitations. An up to date review of the literature was given to the panel members; researchers who were experts on the RAND method performed all the panel processes; the results from the main panel were compared with those from a second panel and, finally, our panel criteria matched most of the criteria found in the literature which supported the criteria validity. As mentioned previously, the RAND method is subject to changes over time. New evidence leads to new indications, contraindications, or better treatment options. Nevertheless, our criteria were designed to pro-

\section{Key messages}

- Updated explicit criteria were developed using the RAND method to evaluate the appropriateness of cholecystectomy.

- The explicit criteria for the appropriateness of cholecystectomy indicate that patients with asymptomatic cholelithiasis, those at high surgical risk, or older patients are more likely to be considered inappropriate for cholecystectomy.

- Symptomatic patients with a low surgical risk or those aged $<70$ years are more likely to be considered appropriate for cholecystectomy.

- The reliability and validity of the explicit criteria were adequate and support their use as a screening tool in quality assurance programmes.

- It is hoped that the use of these explicit criteria will help to reduce inappropriate variations in clinical practice and increase the quality of care for patients with gallbladder disease.

vide clinicians and managers with an up to date review of the literature and evaluation by a group of experts.

In addition to the previous specific limitations of the RAND method, our study presents some more. With the algorithms used we tried to provide the clinician and manager with a tool that might help them in clinical decision making. Nevertheless, the clinical condition of a particular patient can add relevant information not included in our algorithm that may lead to a different decision. To create the indications, categorical variables had to be created. The cut off point of some of them, such as age, could be a source of disagreement. We based our categorizations on the current literature and on the agreement of the whole panel. Our panel had to decide whether or not cholecystectomy was appropriate for a particular patient. We did not ask the panel members to decide between different surgical options currently available such as open cholecystectomy versus laparoscopy, as it is known that asking the panel to make two decisions at once (whether or not to perform an intervention and the type of intervention) creates more confusion. ${ }^{33}$ We performed some validity and reliability studies, although more studies on the validity of the tool could have been performed.

Other groups have developed recommendations using other methods such as consensus groups. ${ }^{34}$ The advantage of our method is that it is more precise and can be applied to individual patients. Nevertheless, this method has not been recommended for that purpose, but as a screening method to determine the degree of appropriateness of one procedure.

Explicit criteria may have different uses-for example, to develop practice guidelines, provide feedback to clinicians, or as a utilization review tool. Some authors ${ }^{35}{ }^{36}$ have suggested that this method may be useful for comparing levels of appropriateness between different patient populations, to study variability, but not for direct care of individual patients. The value of the criteria developed will be judged based on the changes in clinical practice promoted by them, either through the development of clinical guidelines or through other techniques that may contribute to an improvement in the quality of care provided. To be useful this tool has to be viewed as work in progress that needs continuous improvement in response to technological and social changes.

The explicit criteria developed by our panel of experts and the algorithms presented in this study can enhance the quality of patient care in different ways: by using them as practice guidelines; by applying them in real situations, either retrospective or prospectively; to evaluate the appropriateness of clinical practice; or to compare different hospitals or services or improvements over time. The ultimate purpose in all cases is to help to reduce inappropriate variations in clinical practice. 


\section{ACKNOWLEDGEMENTS}

This study was partially supported by a grant from the Fondo de Investigación Sanitaria (98/002-03). Amaia Bilbao received a grant from the Department of Health of the Basque Government. We also thank the following people for their contributions to this study: Drs Aguado, Arenas, Atín, Baile, Casanova, Hinojosa, Lanas, Monés, Pons, Robles, Suárez Alzamora, Valdivieso, and Zaballa. The following also collaborated in parts of the study: Drs Rodríguez Montes, Iturburu, Olabarrios, Sarabia, P Múgica, Gardeazabal, J A Múgica, Bernal, Bujanda, Castiella, Múgica, De las Heras. The authors acknowledge Y Etxeberria and I Lafuente for their contribution to the development of the panel of experts and data introduction.

\section{Authors' affiliations}

J M Quintana, A Bilbao, Unidad de Investigación, Hospital de Galdakao, Galdakao, Vizcaya, Spain

J Cabriada, Servicio de Digestivo, Hospital de Galdakao

I Lopez de Tejada, Servicio de Cirugía, Hospital de San Eloy, Vizcaya, Spain

M Varona, Servicio de Urgencias, Hospital de Basurto, Bilbao, Vizcaya Spain

V Oribe, Servicio de Digestivo, Hospital de Basurto

B Barrios, Servicio de Cirugía, Hospital de Basurto

I Aróstegui, Departamento de Matemática Aplicada, Estadística e Investigación Operativa, Universidad del País Vasco, Lejona, Vizcaya,

Spain

\section{REFERENCES}

1 Bateson MC. Fortnightly review: gallbladder disease. BM 1999;318:1745-8.

2 Lam CM, Murray FE, Cuschieri A. Increased cholecystectomy rate after the introduction of laparoscopic cholecystectomy in Scotland. Gut 1996;38:282-4

3 Johanning JM, Gruenberg JC. The changing face of cholecystectomy. Am Surg 1998;64:643-7.

4 McPherson K, Wennberg JE, Hovind OB, et al. Small-area variations in the use of common surgical procedures: an international comparison of New England, England, and Norway. N Engl J Med 1982;307:13104.

5 Pilpel D, Fraser GM, Kosecoff J, et al. Regional differences in appropriateness of cholecystectomy in a prepaid health insurance system. Public Health Rev 1992;20:61-74

6 Wennberg J. The paradox of appropriate care. JAMA 1987;258:2568-9

7 Dubinsky M, Ferguson JH. Analysis of the National Institutes of Health Medicare coverage assessment. Int J Technol Assess Health Care 1990;6:480-8.

8 Sondenaa K, Nesvik I, Solhaug JH, et al. Randomization to surgery or observation in patients with symptomatic gallbladder stone disease. The problem of evidence-based medicine in clinical practice. Scand J Gastroenterol 1997:32:611-6.

9 Fraser GM, Pilpel D, Hollis S, et al. The agreed indications and contraindications for cholecystectomy. Qual Assur Health Care 1993;5:81-5

10 Friedman GD. Natural history of asymptomatic and symptomatic gallstones. Am J Surg 1993;165:399-404.

11 Boulton RA, Adams DH. Gallbladder polyps: when to wait and when to act. Lancet 1997;349:817.
12 Park RE, Fink A, Brook RH, et al. Physician ratings of appropriate indications for six medical and surgical procedures. Am J Public Health 1986;76:766-72

13 Fraser GM, Pilpel D, Hollis S, et al. Indications for cholecystectomy: the results of a consensus panel approach. Qual Assur Health Care 1993:5:75-80.

14 Scott EA, Black N. Appropriateness of cholecystectomy in the United Kingdom: a consensus panel approach. Gut 1991;32:1066-70.

15 Merrick NJ, Fink A, Park RE, et al. Derivation of clinical indications for carotid endarterectomy by an expert panel. Am J Public Health 1987; 77: 187-90.

16 Rigter H, Meijler AP, McDonnell J, et al. Indications for coronary revascularisation: a Dutch perspective. Heart 1997;77:211-8.

17 Winslow CM, Kosecoff JB, Chassin M, et al. The appropriateness of performing coronary artery bypass surgery. JAMA 1988;260:505-9.

18 Kravitz RL, Laouri M, Kahan JP, et al. Validity of criteria used for detecting underuse of coronary revascularization. JAMA 1995;274:632-8.

19 McKee $M$, Priest $P$, Ginzler $M$, et al. How representative are members of expert panels. Qual Assur Health Care 1991;3:89-94.

20 Coulter I, Adams A, Shekelle P. Impact of varying panel membership on ratings of appropriateness in consensus panels: a comparison of a multiand single disciplinary panel. Health Serv Res 1995;30:577-91.

21 Scott EA, Black N. When does consensus exist in expert panels? J Public Health Med 1991;13:35-9.

22 Phelps CE. The methodologic foundations of studies of the appropriateness of medical care. N Engl J Med 1993;329:1241-5.

23 Brook RH, Chassin MR, Fink A, et al. A method for the detailed assessment of the appropriateness of medical technologies. Int J Technol Assess Health Care 1986;2:53-63.

24 Callaghan J. Twenty-five years of gallbladder surgery in a small rural hospital. Am J Surg 1995;169:313-5.

25 Pessaux $\mathbf{P}$, Tuech JJ, Derouet N, et al. Laparoscopic cholecystectomy in the elderly: a prospective study. Surg Endosc 2000;14:1067-9.

26 Schneider AJL. Assessment of risk factors and surgical outcome. Surg Clin North Am 1983;63:1 113-26.

27 Fleiss JL. Statistical methods for rates and proportions. 2nd ed. New York: Wiley, 1988

28 SAS Institute. SAS procedures guide. Version 6. Cary, NC: SAS Institute, 1994.

29 Kahan JP, Park RE, Leape LL, et al. Variations by specialty in physician ratings of the appropriateness and necessity of indications for procedures. Med Care 1996;34:512-23.

30 Fraser GM, Pilpel D, Kosecoff J, et al. Effect of panel composition on appropriateness ratings. Int J Qual Health Care 1994;6:251-5.

31 Kahn KL, Park RE, Brook RH, et al. The effect of comorbidity on appropriateness ratings for two gastrointestinal procedures. J Clin Epidemiol 1988;41:115-22.

32 Sese TJ, Morlans MG. Acute alithiasic cholecystitis. Medicina Clinica 1985;84:663-6.

33 Quintana JM, Arostegui I, Azkarate J, et al. Use of explicit criteria for total hip joint replacement fixation techniques. Health Policy 2002 (in press).

34 Neugebauer E, Troidl H, Kum CK, et al. The EAES Consensus Development Conferences on laparoscopic cholecystectomy, appendectomy, and hernia repair. Consensus statements, September 1994. The Educational Committee of the European Association for Endoscopic Surgery. Surg Endosc 1995;9:550-63.

35 Shekelle PG, Kahan JP, Bernstein SJ, et al. The reproducibility of a method to identify the overuse and underuse of medical procedures. $N$ Engl J Med 1998;338:1888-95.

36 Naylor CD. What is appropriate care? N Engl J Med 1998;338:1918-20. 